\title{
LA FABRICATION DU CORPS DES « HÔTESSES DE TABLE ». COMMENT L'UNIFORME PRODUIT LES CLASSES DE SEXE DANS LA RESTAURATION
}

\author{
Marie Mathieu
}

Éditions Antipodes | «Nouvelles Questions Féministes »

2019/2 Vol. 38 | pages 16 à 33

ISSN 0248-4951

ISBN 9782889011674

Article disponible en ligne à l'adresse :

https://www.cairn.info/revue-nouvelles-questions-feministes-2019-2-page-16.htm

Distribution électronique Cairn.info pour Éditions Antipodes.

(C) Éditions Antipodes. Tous droits réservés pour tous pays.

La reproduction ou représentation de cet article, notamment par photocopie, n'est autorisée que dans les limites des conditions générales d'utilisation du site ou, le cas échéant, des conditions générales de la licence souscrite par votre établissement. Toute autre reproduction ou représentation, en tout ou partie, sous quelque forme et de quelque manière que ce soit, est interdite sauf accord préalable et écrit de l'éditeur, en dehors des cas prévus par la législation en vigueur en France. Il est précisé que son stockage dans une base de données est également interdit. 


\title{
Grand an
}

\section{La fabrication du corps des « hôtesses de table". Comment l'uniforme produit les classes de sexe dans la restauration}

\author{
Marie Mathieu
}

À l'occasion d'un emploi alimentaire dans un restaurant d'une chaîne française, dans un quartier populaire de Paris, j'ai constaté que la mise en conformité des corps aux règles édictées par la société-employeur était plus exigeante, plus coûteuse et plus éprouvante pour les serveuses que pour les serveurs, désigné e.s respectivement par l'entreprise "hôtesses et hôtes de table " ${ }^{1}$. Ces règles définissent l'uniforme attribué aux unes et aux autres et les normes esthétiques qu'il leur faut respecter. En effet, en France, aucune loi n'empêche une entreprise d'imposer à ses salarié.e's un code vestimentaire. Mais si l'article L1121-1 du Code du travail stipule que "nul ne peut apporter aux droits des personnes et aux libertés individuelles et collectives de restrictions qui ne seraient pas justifiées par la nature de la tâche à accomplir ni proportionnées au but recherché", son interprétation laisse un flou quant à la légitimité des règles esthétiques et vestimentaires formulées par les entreprises. Ce flou m'a paru constituer un cadre propice à une étude de la construction des corps sexués au travail. Sociologue de formation et ancrée dans une perspective féministe matérialiste, je me suis particulièrement intéressée au travail esthétique, gratuit et invisible, qu'implique le "look" attendu des employé'e-s de salle du restaurant Steaky ${ }^{2}$ - un travail exigé avant tout des femmes. Au cours des six mois durant lesquels j'ai travaillé dans cet établissement en tant qu'hôtesse de table, j'ai fait un recueil systématique de mes observations, des informations que mes 
La fabrication du corps des "hôtesses de table".

Comment l'uniforme produit les classes de sexe dans la restauration

Marie Mathieu

collègues m'ont fournies lors de nos échanges durant nos sorties et dans les vestiaires et de différents documents officiels produits par l'entreprise, dans le but d'écrire un article - une fois cet emploi terminé - qui témoignerait du travail des serveuses et qui montrerait comment le façonnage des corps au travail participe de la production de la classe des femmes.

Ma démarche resserre la focale sur les serveuses, même si je les compare à certains moments aux serveurs, ne serait-ce que pour situer le cadre genré dans lequel elles travaillent. Ce choix tient à plusieurs facteurs : elles sont plus nombreuses que les serveurs, les postes d'encadrement sont majoritairement occupés par des hommes, ma propre position de serveuse m'a permis d'être au plus près de ce qu'impliquent leur travail d'hôtesse de table et leur statut de femmes. Enfin, il m'a paru que le travail esthétique qu'elles fournissent pour répondre aux attentes de l'employeur se déroule dans des conditions révélatrices de la valeur accordée au travail de très nombreuses femmes dans les services et que, pourtant, il est rarement étudié. En effet, si le corps est l'objet d'un regain d'intérêt de la sociologie du travail depuis une vingtaine d'années (Jacquot et Volery, 2019) et que les habits de travail ont fait l'objet d'articles l'abordant notamment à l'aune du genre (Rennes, Lemarchant et Bernard, 2019), si les différentes formes que prend le travail gratuit et invisible sont au cœur de nouvelles analyses (Simonet, 2018; Robert et Toupin, 2018), peu de recherches ont analysé le travail invisible de production des apparences professionnelles dans les emplois de service et le coût qu'implique pour de nombreuses salariées l'uniforme au féminin. Pourtant, il participe de la pénibilité de ces emplois et permet d'appréhender la manière dont le travail salarié s'immisce jusque dans les espaces considérés les plus privés, tels que les salles de bains.

À partir de l'analyse des consignes concernant la présentation physique des salarié·e's du restaurant, inscrites dans des documents et des affiches édictant les "bonnes" pratiques en termes d'habillement et d'allure, cet article questionne dans un premier temps le caractère genré des modèles diffusés par l'entreprise au personnel. De manière à saisir les caractéristiques de l'uniforme au féminin, les attentes normatives de la chaîne envers les femmes travaillant en salle sont analysées selon l'échelon qu'elles occupent dans l'organigramme de l'entreprise. La seconde partie est centrée sur le travail des hôtesses de table et les dépenses qu'entraînent pour elles l'ensemble de ces prescriptions. À travers l'analyse de leurs discours et des rappels à l'ordre formulés par les supérieurs hiérarchiques, j'illustre la division sexuée du travail corporel tout comme la charge différenciée qu'il recouvre selon les employées et certaines formes de micro-résistances individuelles face à ces règles régissant leurs corps. Enfın, je montre comment certaines caractéristiques de l'uniforme des serveuses (l'exposition publique et directe d'une grande partie de leur corps et le port de chaussures à talons) renforcent la pénibilité de leur travail et les inégalités de genre. Afin d'y faire face, les femmes de l'établissement s'entraident parfois les unes les 
autres et développent des stratégies pour soustraire certaines zones de leurs corps aux regards de la clientèle, préserver leur intimité, leur pudeur et leur santé physique - stratégies que cette dernière partie de l'article examine.

\section{Des uniformes « créateur[s] d'équipes ${ }^{3}$ et marqueurs du genre}

Suite à son entretien d'embauche, tout·e nouvel-le employé·e du restaurant étudié se voit remettre un feuillet de 26 pages dit "Livret d'accueil " dans lequel sont formalisées les normes du "look" que les salarié·e·s doivent adopter. Ce livret ainsi qu'un poster rappelant les bonnes pratiques en matière de look (poster que je reproduis plus loin) représentent donc d'excellents outils pour analyser les exigences vestimentaires et corporelles de Steaky à l'égard des employé·e·s, l'importance attribuée à l'apparence physique selon les catégories socioprofessionnelles et le travail esthétique attendu des serveuses.

\section{Le Look Steaky: une préoccupation avant tout à l'égard des employées}

Durant les six mois où j'ai travaillé dans ce restaurant, chacun·e de mes collègues en salle, soit douze hôtesses de table, cinq hôtes de table/barmen et cinq commis de salle ${ }^{4}$, a eu en mains le livret d'accueil énonçant les prérogatives vestimentaires et corporelles liées à son contrat de travail.

Le "Look» attendu du personnel recouvre trois pages complètes du livret, auxquelles s'ajoutent une page dédiée aux "Comportements \& Attitudes" et un ensemble de commentaires épars sur la tenue physique des employé·e·s. Le livret défınit ainsi la mission des serveurses et serveurs: "Votre rôle: représenter notre style et notre image de marque, l'hôte(sse) de table STEAKY est notre témoin auprès des clients ", après avoir présenté le script de la prise en charge des client·es: :Tout commence avec l'hôtesse d'accueil : une main qui ouvre la porte, un sourire, une attention particulière pour chaque client... Puis le barman donne le tempo [...]" ${ }^{5}$. C'est donc avant tout aux membres de l'équipe de salle que ce document s'adresse et notamment aux femmes, comme

3. Je reprends ici l'expression mentionnée sur l'étiquette de la robe constituant l'uniforme des hôtesses de table.

4. Deux de ces commis étaient en contrat à durée indéterminée (un homme et une femme) et trois étaient intérimaires (deux hommes et une femme).

5. Dans cet enchaînement-type, les employés travaillant en cuisine sont absents. Cela illustre l'invisibilité plus générale des salariés racisés des cuisines - pendant mes six mois chez Steaky, c'était très majoritairement des hommes d'origine malienne et sénégalaise - qui, en dehors des temps dédiés au repas du personnel, ne peuvent circuler dans la salle du restaurant et demeurent cachés de la vue des client·e·s durant leurs heures de travail. En ce sens, ce groupe est constitué par les normes de l'entreprise comme symétriquement opposé à celui des serveuses.

18. | NQF Vol. 38, №2 / 2019 
La fabrication du corps des "hôtesses de table".

Comment l'uniforme produit les classes de sexe dans la restauration

Marie Mathieu

le confirme l'ordonnancement des pages où les consignes destinées aux "Hôtesses de Table» précèdent celles à l'attention des «Hôtes de Table \& Barmen" et des "Cuisiniers". Aussi, la page "Look" pour les hôtesses de table contient 902 signes et se découpe en trois sous-sections: "Le corps", "La tenue" et "Les accessoires". Dans la première, ce ne sont pas moins de six éléments du corps des femmes qui sont traités sous les intitulés: "le regard», "le sourire", "les mains", "la coiffure», "les collants" et "les pieds". Ces sous-sections contrastent avec les "règles de base à l'élégance" que doivent respecter les hôtes de table/barmen formalisées en 514 signes. Chez Steaky, tandis que les serveurs doivent être "rasé[s] de près", les serveuses sont contraintes de maquiller leurs yeux, joues, lèvres et ongles selon des règles extrêmement précises: "Trait de crayon ou eye-liner, blush sur les joues et fard à paupières. [...] Rouge à lèvres (il est possible de mettre tout type de rouge en accord avec le vernis). [...] Vernis à ongles (il est possible de mettre tout type de vernis sauf transparent, vert, bleu, jaune, noir)». Le poster affiché dans les coulisses du restaurant à l'adresse du personnel de service précise que ce vernis doit être "de préférence rouge».

Ce catalogage des couleurs du vernis effectué par l'entreprise suggère un modèle de l'élégance au féminin marqué par la classe sociale. En effet, si les vernis vert, bleu, jaune ou noir sont proscrits, associés à une féminité populaire, le vernis transparent d'usage dans la restauration de luxe est lui aussi banni. L'absence de vernis pour sa part est interdite, même si certains restaurants gastronomiques l'encouragent par mesure d'hygiène, les mains étant parfois en contact avec les aliments dans les assiettes. Pour répondre au modèle de féminité prôné par Steaky, les serveuses doivent aussi avoir une coiffure "nette et élégante», porter des collants "chair en été - noirs en hiver", avoir des "chaussures noires, unies, fermées, type escarpin avec des talons de $3 \mathrm{~cm}$ minimum " (non compensées selon les illustrations de chaussures associées au texte) et porter des bijoux (bagues, colliers, bracelets, boucles d'oreilles). Alors que "les piercings sont interdits" (voir le poster), les boucles d'oreilles des serveuses doivent "se voir", les obligeant ainsi à afficher ostensiblement cette marque symbolique d'une socialisation au féminin (Monjaret, 2005 : 123 ; Ciambelli, 1996 ; Verdier, 1979 : 192, 205 ; Guillaumin, [1992] $2016: 116)$.

Le travail de représentation/figuration qu'effectue le groupe des serveuses est primordial pour le restaurant, comme le suggère l'intitulé de leur poste: "hôtesse". Pour autant, passer de "serveuse" à "hôtesse de table" est un changement d'appellation qui n'a nullement conduit, comme l'a montré Philippe Alonzo (1998) pour les caissières de la grande distribution, à une diversification ou à un enrichissement du travail, cette dénomination rappelle tout au plus le prestige autrefois conféré à l'hôtesse de l'air (Perramond, 2015; Baum, 2012) - un prestige inextricablement lié au port d'un uniforme et à un capital corporel (Hidri Neys, 2013). Alors que le titre d'hôtesse tend justement aujourd'hui à être délaissé dans le transport aérien au profit de celui de 


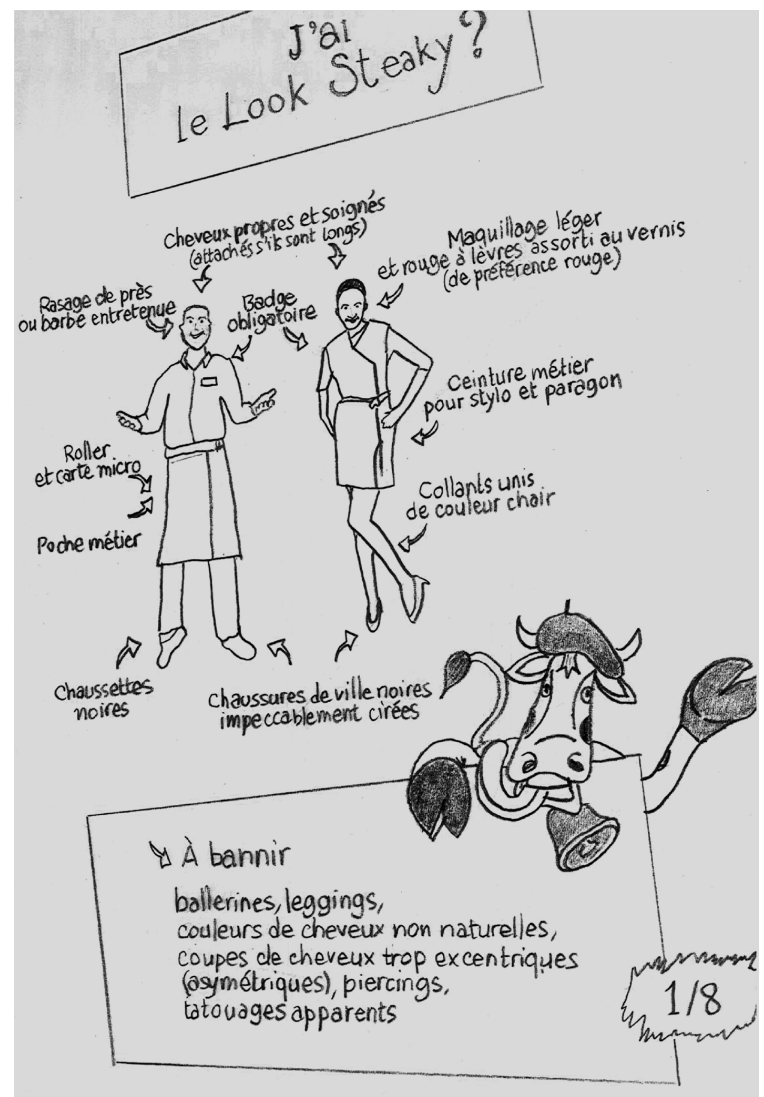

Poster du Look Steaky exposé (parmi 8 autres) dans un espace réservé au personnel de salle (seuls le nom et le logo de l'entreprise ont été modifiés, par souci d'anonymisation).

personnel navigant de cabine, pour se détacher des rôles stéréotypés d'hôtesses de l'air et de stewards (Barnier, 2014 : 134), il s'est imposé progressivement dans les différents métiers de service: hôtesses de caisse (Alonzo, 1998 ; Benquet, 2013) et/ou d'accueil (Schütz, 2006, 2018 ; Hidri Neys, 2013), hôtesses de vente (Hidri et Louveau, 2005) et hôtesses de table. Ce titre rend compte en lui-même de l'importance que les sociétés-employeurs attachent à la présentation de soi dans ces différents métiers fortement féminisés, ce qui conduit, on l'a vu avec l'analyse des documents de Steaky, à une hyper-codification du corps des femmes employées dans les services.

\section{Les attributs du pouvoir au féminin}

Dans la salle du restaurant étudié se côtoient des employées ayant des fonctions différentes. Les hôtesses de table, qui composent le plus gros des effectifs, assurent la mise en place pour le service, l'installation des client·e.s, la prise de commande et sa transmission, le service, le débarrassage, la facturation, l'encaissement, le nettoyage et le redressage des tables, le remplissage et 
le ménage de leur console, "leur caisse ${ }^{6}$ et la "remontée-cave» ${ }^{7}$ le soir. Elles sont encadrées par la «leader», leur supérieure hiérarchique directe chargée de la formation des nouvelles recrues, de la fabrication des plannings, de la répartition des rangs entre hôte-sse's de table, et de faire en sorte que le personnel de salle respecte les règles de l'entreprise et que le service fonctionne bien. De plus, la leader doit vérifier les montants des caisses fournies par les serveuses et serveurs. Enfin, se situant au-dessus de la leader dans l'organisation hiérarchique de l'entreprise, la directrice adjointe - fonction occupée le plus souvent par un homme ${ }^{8}$ - est responsable du recrutement des nouveaux serveurs et serveuses. Contrairement à la leader, elle ne fait pas de formation et est donc moins souvent amenée à faire du service en tant que tel. Mais pour les autres tâches, toutes deux se relaient et travaillent en alternance.

Selon leur position dans l'organigramme du restaurant, les employées n'ont pas les mêmes tenues, ce qui permet de les distinguer d'un simple coup d'œil. À Steaky, chaque hôtesse de table doit porter une tenue bicolore noire et marron composée d'un tee-shirt à manches trois-quarts, une robe - sans poche -, un badge où figure son prénom orné du personnage enseigne de la chaîne de restaurant, des escarpins noirs à talons et des collants chair. En effet, si le livret d'accueil signale que les serveuses doivent porter des collants "noirs en hiver», sous prétexte d'harmonie des couleurs, ceux-ci sont proscrits dans l'établissement où j'ai travaillé, quelle que soit la saison. L'uniforme des serveuses est complété par une "ceinture métier" attachée autour de leur taille. Cette ceinture est équipée de petites poches, leur permettant de ranger stylo, carnet de commande et limonadier. Elle se substitue au tablier qu'ont les serveurs, sans pour autant l'équivaloir: en effet, le tablier des serveurs est doté d'une grande poche particulièrement pratique pour mettre davantage d'outils de travail, notamment la caisse, ainsi que des effets personnels comme le téléphone portable ou le portefeuille, ce que limitent beaucoup plus les petites poches de la ceinture des serveuses. Le tablier des serveurs, de même que les poches profondes de leurs pantalons, illustrent par effet miroir le monopole masculin des outils mis en lumière par Paola Tabet (1998 : 75). Car les serveuses, elles, sont contraintes de laisser leurs affaires personnelles dans un tiroir et surtout d'y laisser leur caisse, ce qui les conduit à faire de multiples pas supplémentaires pour encaisser les consommations de la clientèle.

Bien que vêtue d'un uniforme aux teintes identiques et exposant elle aussi son identité via le port d'un badge prénom, la leader que les serveuses et serveurs appellent "Dame Renée» porte pour sa part une chemise - avec

6. Faire sa caisse implique, après chaque service, d'imprimer le bilan détaillé de ses encaissements à partir du logiciel de caisse, d'organiser ces derniers selon les modes de paiement de la clientèle et de s'assurer de leur correspondance avec les montants que le logiciel indique, après avoir soustrait son fond de caisse.

7. Cette étape consiste à décompter les boissons vendues dans la journée et à remonter de la cave les bouteilles manquantes pour remplir la réserve de boissons située au premier étage du restaurant et les frigidaires du bar.

8. Durant toute la période de mon emploi, trois hommes et une femme se sont succédé à ce poste. 
un col couvrant sa nuque - sous un gilet sans manches, des collants chair et une jupe légèrement plus longue que celle des hôtesses de table. Contrairement à ces dernières, elle bénéficie d'un haut à manches longues, retroussées à mi-bras et retenues par des languettes boutonnées, mais pouvant être déroulées. Par ailleurs, si Dame Renée n'a pas de ceinture métier, elle possède dans son gilet une paire de poches lui permettant de ranger ses ustensiles de travail (les mêmes que ceux des serveuses) mais aussi certains effets personnels (dont son téléphone portable).

La directrice adjointe quant à elle porte un uniforme qui ne lui est pas fourni par l'entreprise et qui la distingue clairement des autres employées. Bien qu'astreinte au port d'un tailleur foncé et d'une chemise claire, elle bénéficie d'une plus grande liberté quant aux modèles et couleurs de ses tenues. De plus, elle n'est pas obligée d'exposer son identité à travers le port d'un badge et elle est l'unique employée de la salle du restaurant à être appelée par son nom de famille précédé du titre "Madame». Enfın, elle peut déroger à deux règles contraignant les tenues féminines : l'exposition des jambes et des avant-bras. En effet, dans le personnel, c'est la seule femme à pouvoir mettre des pantalons et revêtir une veste. Cette pièce de son uniforme lui permet d'avoir deux poches (en plus de ses deux poches de pantalon) et donc de pouvoir garder sur elle ses instruments de travail et quelques objets personnels.

Ces caractéristiques de la tenue de la directrice adjointe, comparées à celles des uniformes des serveuses et de l'ensemble du personnel masculin, suggèrent que les femmes ne peuvent accéder à certains privilèges vestimentaires qu'en gravissant les échelons. Cette montée en grade n'est pas nécessaire pour les hommes: quel que soit leur statut dans le restaurant, ils (commis de salle comme directeur) portent un pantalon avec des poches internes et une chemise à manches longues, recouvrant leurs avant-bras et leur nuque. Mais elle l'est pour les employées et de fait, plus elles s'élèvent dans la hiérarchie du restaurant, plus leur uniforme s'approche de celui porté par les hommes. Elles peuvent alors exercer leur fonction d'encadrement en usant des prérogatives préférentiellement accordées aux hommes, mais sans pour autant, en tant que femmes, se démettre de certains codes féminins : même aux rangs les plus élevés, elles sont contraintes de porter des vêtements cintrés ou serrés (marquant leur morphologie), des chaussures type escarpins à talons et de se maquiller le visage et les ongles.

Ces différences de genre se voient à tous les niveaux hiérarchiques. Même si l'uniforme de la directrice adjointe se rapproche plus de l'uniforme masculin que celui d'une hôtesse de table et d'une leader, sa tenue diffère clairement de celle du directeur adjoint. Outre les contraintes que je viens de citer (comme les chaussures à talons ou le maquillage), la directrice adjointe porte en effet un pantalon plus serré que ses prédécesseurs et son successeur hommes. De plus, ses poches, bien que presque aussi nombreuses - les vestes pour femmes, contrairement à celles des hommes, n'ont pas de poche intérieure -, 
sont moins grandes. Cela n'a bien entendu rien de spécifique à l'entreprise étudiée: de manière générale, les vêtements destinés aux femmes sont plus souvent dénués de poche (ou ont de "fausses poches»), tandis que leur déclinaison masculine en est dotée. Et lorsque les vêtements féminins ont des poches, celles-ci sont toujours moins profondes que celles observées dans de mêmes habits destinés aux hommes.

Les tenues vestimentaires des supérieures travaillant chez Steaky sont donc hybrides: elles restent marquées du sceau de la féminité tout en arborant certains privilèges des tenues masculines. Au sein de l'équipe de salle, les uniformes combinent genre et position hiérarchique dans l'organigramme. On peut y identifier deux idéaux-types de tenues de travail s'opposant en tous points. Tandis que le directeur adjoint porte une chemise à col, une cravate, un pantalon et une veste, possédant ainsi plus de poches que toute autre catégorie d'employé·e·s, le groupe des hôtesses de salle a un uniforme caractérisé par l'absence de poches et par la proportion importante de chair qu’il dévoile. Si chaque échelon hiérarchique est marqué par des différences de genre, on observe une masculinisation croissante de l'apparence des femmes en passant vers le haut de l'organigramme et donc une masculinisation associée au pouvoir. Cependant, celle-ci n'efface que pour partie les prescriptions liées aux tenues des employées et les figures exemplaires mises en valeur par l'entreprise participent à tous les niveaux de la "réassurance des identités hétérosexuées" (Schütz, 2006:143).

\section{De la production du corps des serveuses à la production sociale de classes}

Si les uniformes genrés renforcent l'identification des groupes travaillant chez Steaky, c'est la division sexuée du travail de service qui donne corps à la hiérarchie des catégories socioprofessionnelles, comme l'ont montré les quelques enquêtes qui l'analysent dans les restaurants ou les bars (Mann et Spradley, 1979 ; Fellay, 2010). Dans l'établissement étudié, tandis que les femmes sont exclusivement assignées à la fonction d'hôtesse, les employés peuvent se projeter dans des rôles variés (hôte de table, barman, cuisinier) et avoir accès au bar et aux postes de cuisine. Notamment, le contrôle des denrées (de l'alcool et de la nourriture, et plus spécifiquement des viandes, spécialité de la chaîne) s'affiche comme l'apanage des hommes, en charge de leur manipulation, de leur décompte, de leur approvisionnement (au travers des inventaires et commandes) et de leur conservation (via leur tri et l'établissement des "DLC ${ }^{9}$ ). Aux territoires masculins (le bar et les cuisines) s'oppose la salle de restauration, où les hôtesses de table forment la première

9. Acronyme pour " date limite de conservation ». Les DLC sont à inscrire sur les produits entamés et conservés après chaque service. 
ligne face à la clientèle, censées assurer le bon déroulement des repas et la satisfaction des client·e·s. Cette fonction de représentation, qui doit prendre place dans le respect des exigences de Steaky en matière de look, nécessite en amont tout un travail esthétique qui implique une charge de travail et des coûts inégaux entre serveuses et serveurs, ainsi qu'entre employées, soit, en d'autres termes, des inégalités produites respectivement par les rapports sociaux de sexe, d'âge et de race.

\section{Dans les coulisses, le travail et les dépenses invisibles des serveuses}

Avant d' "entrer en scène" dans la salle et après chacun de leur service, les hôte-sse-s de table revêtent et ôtent leur uniforme dans les vestiaires du personnel en sous-sol du restaurant. Selon la loi française, "le temps nécessaire aux opérations d'habillage et de déshabillage, lorsque le port d'une tenue de travail est imposé par des dispositions légales, des stipulations conventionnelles, le règlement intérieur ou le contrat de travail et que l'habillage et le déshabillage doivent être réalisés dans l'entreprise ou sur le lieu de travail, fait l'objet de contreparties. Ces contreparties sont accordées soit sous forme de repos, soit sous forme financière. " ${ }^{10}$ Chez Steaky, ces étapes réalisées en dehors des heures officielles de travail sont rétribuées à travers une prime mentionnée sur le bulletin de paye en tant que "Prime Hab/Deshab ", s'élevant à 1 euro par journée de service. Salarié·e·s à plein temps, ce qui représente, dans l'établissement, 39 heures (a minima) hebdomadaires réparties sur cinq jours, les hôte-sse·s de table perçoivent donc une prime de 5 euros par semaine. Or, cette rétribution très minime fait l'impasse sur au moins deux aspects du travail esthétique fourni dans les coulisses du restaurant. Premièrement, elle ne tient pas compte du fait que les horaires de travail journaliers sont bien souvent coupés, divisés en deux moments séparés par une pause, les employé·e·s assurant ainsi les deux services principaux de la journée (midi et soir). Cette coupure implique que l'habillage et le déshabillage ont lieu deux fois par jour. Deuxièmement, le temps nécessité par ce travail invisible est nettement plus élevé pour les serveuses que pour leurs collègues masculins. En effet, au moment du "dépôt des armes" - expression employée par l'équipe de salle pour désigner la fin d'un service -, les serveurs ne font que détacher leur tablier pour se défaire de l'identité de la chaîne avant de sortir de l'établissement. Sans cet élément, leur uniforme s'apparente à un habit de "civil» ordinaire ${ }^{11}$. En revanche, l'uniforme des serveuses est marqué du sceau de l'entreprise et elles ne veulent pas s'exposer à l'extérieur du restaurant dans cette tenue qui les identifie explicitement à

10. Art. L3121-3 modifié par la loi n²016-1088 du 8 Août 2016-art.8 V.

11. De même pour les directeurs adjoints et le manager (équivalent masculin de la leader), la tenue portée sur le lieu de travail s'apparente à une tenue de " civil ". 
la chaîne et qui voile leur singularité. En outre, leur tenue de travail révèle une grande part de leurs corps. Elles procèdent alors, dans les vestiaires, à un cérémonial quotidien devenu quasi automatique ${ }^{12}$ et qui leur prend du temps.

Ce travail que les hôtesses de table effectuent dans les vestiaires n'est pas rémunéré à hauteur de ce qu'il coûte. Si on estime à 10 minutes ${ }^{13}$ chaque séquence d'habillage ou de déshabillage, une serveuse passe sur une semaine entre $1 \mathrm{~h} 40^{14}$ et, lorsqu'elle a deux journées en coupure, $2 \mathrm{~h} 20^{15}$ à cette tâche, pour une rétribution totale de 5 euros. Sachant que son salaire horaire est de 9,55 euros brut, ces séquences devraient être rémunérées de 15,92 à 22,28 euros par semaine si l'on convertissait ce temps de préparation en temps de travail en salle. La différence ainsi calculée est de 43,68 à 69,10 euros par mois par rapport au salaire que les serveuses perçoivent.

À ceci s'ajoute pour elles un temps quotidien chez soi. Si «le rôle d'hôtesse constitue en partie un prolongement dans la sphère professionnelle du rôle féminin dans la sphère domestique» (Schütz, 2006 : 141), le travail de formatage des corps étend les activités professionnelles de l'apparence à la sphère domestique et remet en question la limite entre espaces-temps professionnel et personnel. La leader, en charge de la formation des serveuses et dépeinte par celles-ci comme un "pur produit Steaky", revient souvent sur l'idée que devenir hôtesse de table dans ce restaurant, c'est comme "apprendre à faire du vélo", c'est-à-dire intérioriser des gestes routinisés qui se prolongent jusque dans l'espace réputé privé des salles de bains. S'y (re)jouent quotidiennement les mêmes actions de mise en conformité du corps avec les prescriptions de la société-employeur: maquillage du visage, manucure et vernissage des ongles (pose et attente pour le séchage), rasage des jambes ou épilation qu'implique le port de collants chair (et teinture capillaire pour certaines, comme je le présenterai par la suite). La (re)construction perpétuelle de ce corps de "vraie femme" selon l'idéologie diffusée par le règlement de Steaky est particulièrement chronophage et exige des serveuses un travail gratuit qui n'est pas sans rappeler l'assignation prioritaire des femmes au travail domestique. Du reste, les pages du livret d'accueil dédiées au look des salarié·e·s présentent la spécificité des uniformes féminins et masculins en l'articulant à la division traditionnelle des tâches domestiques, en particulier de l'entretien du linge (Kaufmann, 1992 ; Champagne, Pailhé et Solaz, 2015): ces pages mentionnent en effet que les tenues des serveurs "sont

12. À trois reprises lors de ce terrain, je me suis surprise à enlever mon uniforme pour le remettre, au lieu d'enfiler mes propres vêtements. Deux autres serveuses m'ont dit avoir connu des épisodes similaires. Ces erreurs signalent l'intériorisation et l'automatisation de ces gestes.

13. Estimation basse pour les femmes.

14. Détail du calcul : 10 min. $x 2$ séquences (habillage et déshabillage) x 5 jours.

15. Détail du calcul : (10 min. $x 2$ séquences $x 3$ jours $)+(10$ min. $x 4$ séquences $x 2$ jours en coupure) 
choisies en fonction de la mode actuelle mais aussi pour leur facilité d'entretien et leur aisance", de même qu'elles signalent aux serveuses que leur tenue «jeune, féminine, élégante, dynamique et fonctionnelle [...] se lave en machine (minimum $30^{\circ}$ ), sèche très rapidement [et] ne nécessite qu'un repassage superflu». Ainsi, le document participe à une réassignation de genre: le nettoyage du linge est un travail "naturellement» (donc non payé) dévolu aux serveuses dans le prolongement des activités associées à leur position de femmes au sein de la famille, tandis que les serveurs sont exemptés de cette tâche domestique jugée dégradante pour eux. Ajoutée au travail esthétique attendu des hôtesses de table, elle révèle une charge inégale de travail en amont (dans les foyers) comme en aval (dans les vestiaires) des heures dites de travail, en défaveur des serveuses.

Ce travail invisible est non seulement extrêmement peu rémunéré, il a aussi un coût financier que les serveuses doivent assumer. Avant même leur embauche, donc pour un essai, les "aspirantes hôtesses de table" doivent acheter tout un matériel : des chaussures noires à talons non compensés de plus de trois centimètres, un collant chair, de grosses boucles d'oreilles, ainsi que des outils de maquillage, spécifiés par le livret d'accueil : fond de teint, blush, eye-liner, fard à paupières, mascara, rouge à lèvres, vernis à ongles (et par conséquent démaquillant, dissolvant et coton). Le coût de cet équipement est assez difficilement quantifiable - d'autant qu'il doit être renouvelé en permanence. Il représente au minimum une dépense de 100 euros pour un simple essai, puis par la suite une dépense d'au moins 40 euros par mois, une serveuse n'utilisant pas moins de 2 à 3 paires de collants par semaine.

En outre, l'obtention de ces éléments nécessite un travail gratuit de consommatrice (comparaison des prix, de la qualité des produits) et un temps de magasinage (dans les boutiques, en amont de l'embauche officielle, puis régulier après signature d'un contrat) qui fait peser sur les serveuses une charge mentale supplémentaire liée à la logistique des achats (notamment l'anticipation des fins de stocks, tout particulièrement des collants).

Enfin, la tenue exigée des serveuses chez Steaky nécessite aussi des savoir-faire plus ou moins maîtrisés selon les femmes. Quelque peu désemparée face à l'écaillement du vernis de mes ongles au travail et au labeur quotidien qu'implique pour moi l'injonction à avoir des ongles colorés et impeccables, j'interroge un jour une collègue qui arbore une manucure toujours parfaite. Elle m'explique se rendre régulièrement dans une "onglerie» pour se faire poser de fausses "griffes" : "Ça te coûte à peu près 10 euros et tu es tranquille pendant au moins trois semaines!» Bien que le recours à des professionnelles (manucure, esthéticienne, coiffeuse) permette aux employées de se soustraire à certaines corvées, il signifie des dépenses et du temps supplémentaires pour pouvoir paraître comme la "bonne" hôtesse de table à laquelle, au bout du compte, toutes les serveuses ressemblent. 
La fabrication du corps des "hôtesses de table".

Comment l'uniforme produit les classes de sexe dans la restauration

Marie Mathieu

\section{Face à l'uniformisation, des différences entre femmes et des micro-résistances}

L'ensemble des prescriptions et proscriptions de l'entreprise sur la tenue des serveuses conduit à la formation d'un groupe en apparence homogène. Ceci est manifeste lorsque les membres de la direction les confondent entre elles, ou lorsque les client·e.s leur prêtent des liens de famille. La plupart des serveuses se voient fréquemment questionnées sur une éventuelle sororité, mais ce phénomène atteint son paroxysme quand nous nous voyons, avec une collègue, suspectées à plusieurs reprises de gémellité.

L'uniformité résulte, on l'a vu, d'une longue liste de devoirs et d'interdits à destination des serveuses, auxquels s'ajoutent de nombreux rappels à l'ordre de la direction. Cependant, dans l'intimité des vestiaires ou dans d'autres moments d'entre-soi, j'ai pu constater, à travers les propos de mes collègues, que la production de ce groupe en apparence homogène suppose un travail esthétique plus ou moins important selon les femmes, leurs corps étant plus ou moins distants du référent de féminité de service exposé et imposé par l'entreprise.

Quel que soit leur âge, les serveuses sont régulièrement appelées par les client.e.s "ma petite" ou "mademoiselle" et lors d'une sortie entre serveuses, Stéphanie, une toute nouvelle hôtesse de table, souligne qu'en uniforme, nous semblons "toutes plus jeunes" que dans nos propres vêtements. Pourtant, paraître jeune implique une charge de travail et des dépenses différentes selon les femmes. Suite à une remarque du directeur adjoint sur ses racines blanches apparentes, Dame Renée - la plus âgée des employées en salle - me confie: "Quand est-ce que j'ai le temps d'aller chez le coiffeur?» Du fait d'un sous-effectif permanent et en l'absence d'une direction adjointe stable, Dame Renée est très présente sur le site. Il lui arrive même de dormir à l'hôtel adjacent, en accord avec la direction, pour ne pas perdre de temps de sommeil en rentrant chez elle. Je lui soumets l'idée de garder sa coupe au carré mais en retrouvant sa couleur naturelle blanche. Cette idée semble la séduire. Toutefois, au retour de son repos, elle a une nouvelle coloration châtain et m'explique que «les cheveux blancs ne passeraient sans doute pas au niveau de la direction". Autrement dit, si le poster de rappel du Look Steaky mentionne, parmi les éléments "à bannir" pour toutes et tous, "les coupes de cheveux trop excentriques (asymétriques)" et "les couleurs de cheveux non naturelles", le blanc semble exclu de cette dernière catégorie, contraignant les employées concernées à une opération régulière de coloration, ce qui participe de la construction sociale de classes d'âge au sein du groupe des femmes.

Rien n'est mentionné sur les lunettes de vue dans les documents diffusés par la chaîne, mais l'ensemble des images illustrant le Look Steaky n'en montre pas. Nous sommes deux serveuses à en porter et sommes à plusieurs 
reprises encouragées par le directeur adjoint à mettre des lentilles de contact. Ainsi, les lunettes ne font pas partie de la panoplie des hôtesses car elles donnent à voir un corps "défaillant" contrastant avec l'image de jeunesse qu'elles doivent incarner et qui conforte le rapport de domination des cliente.es sur la prestataire. Les lunettes - comme tout indicateur de vieillissement - pourraient créer une confusion dans le "rapport serviciel " (Jeantet, 2003), d'autant plus que cet accessoire peut être vu comme un symbole de capital culturel dont les serveuses, selon les représentations communes, seraient dépourvues (Laperrière, Messing et Bourbonnais, 2010 : 43).

Certaines serveuses témoignent d'une charge de travail quotidienne plus lourde pour correspondre au modèle de la chaîne. Ainsi, Francesca s'esclaffe lorsque nous évoquons l'épilation des jambes qu'implique le port de collants chair. Elle ajoute: "Avec une pilosité de "Portugaise", c'est quand même un peu plus la galère!», signalant un investissement différent entre les femmes ayant des jambes à la pilosité dense et brune et celles ayant un léger duvet clair. En outre, pour elle, cette charge supplémentaire se cumule à une autre. Bien qu'elle se plie quotidiennement à un travail de mise en conformité de son corps avec les règles de l'entreprise et réunisse proprement sa chevelure dans une queue-de-cheval, elle est sommée, lors d'un rendez-vous avec l'équipe de direction, de remédier au "laisser-aller" dont elle aurait récemment fait preuve, notamment en négligeant sa coiffure. Lorsque Francesca nous rejoint ensuite, elle dit avec colère: "Mes cheveux, j'y peux rien, ils sont comme ça!» Est ainsi exigé d'elle un travail supplémentaire de "dressage" de son épaisse et longue chevelure noire et ondulée. Pour obtenir une coiffure plus "nette», elle est contrainte de lisser ses cheveux (et d'acheter des produits cosmétiques supplémentaires permettant une domestication plus efficace de sa chevelure), à l'instar de la directrice adjointe dont la longue chevelure noire très épaisse, affichée dans une queue-de-cheval d'une raideur parfaite, incarne l'exemple. Tandis que Francesca est régulièrement réprimandée sur la manière dont elle s'occupe de sa coiffure, Lucie, une serveuse aux cheveux mi-longs blonds et fins, en est épargnée alors même qu'elle travaille bien souvent les cheveux détachés.

Ainsi, la mise en conformité des corps (ici la pilosité et les cheveux) avec les modèles de la chaîne exige des serveuses un travail inégal qui participe à la construction sociale de groupes, notamment racisés, au sein même de la classe des femmes.

Cependant, certaines serveuses résistent à ces prescriptions, à l'instar de Juliette. Malgré de nombreux blâmes, elle se soustrait à la règle selon laquelle les hôtesses de table chez Steaky doivent avoir de grosses boucles d'oreilles, la considérant "stupide». Lors d'une réunion amicale, elle nous dit: «Et qu'est-ce qu'on fait quand on n'a pas les oreilles percées? C'est comme si toutes les femmes devaient avoir des trous dans les oreilles. Je ne vais pas me faire percer les oreilles pour eux non plus!» D'ailleurs, face au 
reproche du directeur adjoint sur son teint trop pâle, Juliette réagit avec colère: "Je ne tolère pas le maquillage. J'ai plein d'allergies aux produits. Il n'y a que la poudre Chanel qui coûte une fortune et j'vais pas en mettre une tonne. Et puis, si j'en mets une tonne j'aurai le teint plus foncé que les mains! Ça paraîtra bizarre!»

\section{Derrière les apparences...}

Malgré ces différences au sein de la classe des femmes, toutes les serveuses s'accordent sur la gêne occasionnée par deux éléments de l'uniforme au féminin : d'une part, elles estiment que leur corps est trop dévoilé et que cela ne se justifie pas; d'autre part, elles se plaignent du fait que le port de chaussures noires avec des talons de trois centimètres minimum non compensés les handicape dans la réalisation de leur métier. La sexualisation du corps et le port de chaussures à talons sont donc deux éléments centraux dans la construction sociale des corps sexués (Guillaumin, [1992] 2016) qui rendent le travail des serveuses particulièrement pénible.

\section{Des corps de femmes exposés}

L'élément le plus frappant de la comparaison des déclinaisons féminine et masculine des uniformes de l'équipe de la salle est la différence de surface de chair exposée: les serveuses exposent leurs avant-bras, leur nuque, leur décolleté et surtout leurs jambes puisqu'elles portent des robes courtes.

Ce dernier détail est très important parce que les serveuses sont postées en priorité au rez-de-chaussée du restaurant, zone par laquelle les client·e·s accèdent à l'ensemble des salles de l'établissement, mais aussi seule section visible de l'extérieur aux passant·e·s (elle a de grandes baies vitrées). "Mises en vitrine" sous un contrôle redoublé, les serveuses sont contraintes de monter et descendre en permanence un grand escalier central aux garde-corps latéraux transparents, pour prendre les plats en cuisine à l'étage et les déposer en salle au rez-de-chaussée, tout comme pour remonter à la plonge les plats débarrassés. Il existe bien un monte-charge, mais il dysfonctionne en permanence ${ }^{16}$.

Dès mon premier jour de travail, Olivia m'explique que certains clients ont pour habitude de s'installer à des tables précises pour "mater" sous les robes des serveuses quand elles utilisent l'escalier. D'ailleurs, certains ne s'en cachent pas, comme le raconte Juliette à propos d'un client qui s'est permis de lui dire avec un air amusé : "J'aime vous voir monter et descendre les escaliers." La robe courte des serveuses et l'interdiction du port de

16. Le monte-charge n’a jamais été réparé durant la totalité de ma période de travail. 
collants foncés ou de leggings (voir le poster) les contraignent à exposer des parties de leur corps considérées comme intimes et favorisent ce type de voyeurisme. Dans les faits, seule une serveuse est autorisée à mettre un legging au travail. Dès l'annonce de sa grossesse alors déjà visible, Olivia a en effet pu couvrir ses jambes (tout comme la directrice adjointe), rejoignant le groupe des "femmes respectables». Tout comme la montée en grade, la gestation et le statut de mère en devenir permettent de se soustraire à cette règle d'exposition des jambes imposée à l'ensemble des serveuses et donc d'échapper aux conséquences qui s'ensuivent.

Aussi, les hôtesses de table se plaignent du froid auquel leur "nudité» les expose. Par exemple, lorsque Kevin, serveur, dit avoir trop chaud à cause de sa chemise, Juliette rebondit: "Ben ouais mais moi je préférerais avoir trop chaud de temps en temps plutôt que d'avoir trop froid tout le temps, et d'être perchée sur des talons en collants! J'ai même un ongle [à l'orteil] qui est tombé.» ${ }^{17}$

À travers ces propos se donne à voir la manière dont les corps des femmes sont affichés, mais aussi physiquement éprouvés. Si certaines hôtesses ont des allergies aux produits constituant la trousse de maquillage obligatoire, se plaignent de la constante sensation de froid ou de brûlures des mains et avantbras liées au port des assiettes, les chaussures à talons de plus de trois centimètres non compensés sont la partie de l'uniforme qu'elles critiquent le plus, du fait qu'elles sont obligées de rester debout pendant des plages horaires pouvant durer huit heures, de marcher, monter et descendre des escaliers à longueur de journée, les bras souvent chargés d'assiettes ou de plateaux de verres. Réel "handicap» dans la réalisation de leurs tâches, ces chaussures contraignent les corps des femmes, causent de multiples douleurs musculaires, articulaires, épidermiques (Ruault, 2010). Après quatre mois de travail à plein temps, il m'est douloureux de marcher avec des chaussures plates durant mes jours de repos et je ne compte plus mes ampoules aux pieds. Ainsi, le port de chaussures à talons implique que les serveuses achètent des pansements et des produits pour réparer leurs pieds blessés, mais aussi de multiples chaussures pour trouver la paire qui leur procurera le moins d'inconfort - des chaussures qui coûtent particulièrement cher. À titre d'exemple, il m'a fallu acheter deux paires de chaussures avant de me décider à investir plus de 90 euros dans une marque réputée pour le confort qu'elle offre.

La construction genrée des corps dans l'entreprise par les uniformes place donc les serveuses devant des injonctions contradictoires: elles doivent se déplacer davantage que les serveurs du fait que, on l'a vu précédemment, leur tenue de travail n'offre pas de poches suffisantes pour garder sur elles tous leurs instruments de travail (notamment leur caisse), tout en devant porter un type de chaussures qui leur crée un inconfort et des maux supplémentaires.

17. Durant mes six mois de travail, nous sommes trois serveuses à vivre cette expérience. 
En outre, le travail esthétique qu'elles doivent fournir en amont et en aval des séquences en salle leur coûte plus cher en temps et en argent que cela n'est le cas pour la classe des hommes. Enfin, l'activité de service semble impliquer également un travail émotionnel (Hochschild, [1983] 2017) supplémentaire pour les serveuses : quand le livret d'accueil recommande au personnel d' "avoir toujours le sourire", il s'adresse avant tout aux femmes - comme dans l'ensemble des restaurants (Hall, 1993 : 460). Les sourires attendus des serveuses correspondent à un réel et permanent effort pour dissimuler leurs vraies émotions, leurs douleurs, leur gêne et leur inconfort. Mais à défaut de pouvoir laisser transparaître leur malaise, les employées de Steaky s'entraident pour l'amenuiser.

\section{Transmission des ficelles du métier}

Majoritairement non syndiquées ${ }^{18}$ et fortement divisées par le renouvellement fréquent du personnel et par l'organisation du travail, les hôtesses de table - et plus largement les femmes employées pour travailler dans la salle du restaurant observé - se soutiennent cependant les unes les autres et s'entraident à maintes occasions. Cela se traduit surtout par des échanges de "tuyaux" et de "ficelles" pour économiser le temps et l'énergie dédiés à la mise en conformité des corps de femmes au modèle prescrit par l'entreprise, ainsi que pour s'aménager un peu plus de confort.

En premier lieu, les marques et magasins de chaussures sont des données précieuses que les plus anciennes communiquent aux plus jeunes. À cela s'ajoute la circulation entre employées de techniques pour dissimuler la plus grande part de chair sans enfreindre le code vestimentaire de Steaky. Par exemple, lors de ma rencontre avec la directrice adjointe, elle me conseille d'opter pour une taille d'uniforme au-dessus de celle que j'utilise habituellement car "les robes sont très courtes!", ce que je fais alors. Mais me sentant encore très dénudée, la leader me conseille de faire revoir l'ourlet par une couturière de manière à augmenter la longueur de tissu couvrant mes jambes. Cette stratégie sera toutefois à mes frais et en dépit du fait que son conseil m'a permis de gagner quelques centimètres m'exposant moins, mon malaise en circulant sur l'escalier ne s'est pas dissipé. C'est Olivia, la serveuse enceinte dont j'ai parlé auparavant, qui trouve la solution: elle m'offre un de ses shortys noirs à enfiler par-dessus mes collants.

Ces échanges entre employées constituent donc des stratégies qui permettent de préserver son intégrité au travail. Elles permettent notamment de résister à la sexualisation du corps, comme en témoigne l'histoire de ce

18. Bien que travaillant à plein temps pour l'entreprise et depuis plusieurs mois, la plupart des serveuses et serveurs considèrent leur emploi comme temporaire. 
fameux escalier que Dame Renée me raconta un soir: "Autrefois, les entremarches étaient des espaces ouverts; ce n'est que peu de temps avant mon embauche que les marches ont été fermées. Cette modification est survenue à la suite de la demande d'une ancienne leader qui avait dit à la direction: "Les filles de mon équipe ne sont pas des putes non plus !" "

\section{Conclusion}

Les employées de Steaky font souvent référence à la prostitution, notamment à propos des contraintes vestimentaires et corporelles que l'entreprise leur impose. Ainsi, Juliette nous rapporte qu'elle a été rappelée à l'ordre par le directeur adjoint au sujet du maquillage de son visage et ajoute: "S'il me voyait au réveil, il verrait déjà tout le temps qu'il me faut pour avoir cette tête-là. J'ai passé deux heures pour avoir cette tête. [...] Je n'ai pas envie de me maquiller comme une pute!» Tout en soulignant le temps (non salarié) que suppose le maquillage des serveuses, elle évoque la sexualisation qu'il signifie. En effet, les normes corporelles qui leur sont imposées, de même que la manière dont elles sont mises en scène dans l'espace du restaurant, participent d'une exposition et d'une sexualisation de leurs corps et contribuent, tout comme la consommation de viande promue par la chaîne (Desaulniers, 2018), à la réassurance d'une masculinité hégémonique (Connell, 2014 : 74-75). D’ailleurs, le livret d'accueil les défınit comme "ambassadrices de charme", une expression qui souligne bien qu'au-delà du service d'un bon repas, les serveuses se doivent de travailler leur corps et leurs émotions et faire fi de la pénibilité du travail pour être désirables et donc séduire. Comme le relevait Lisa Adkins (1992 : 218-219) : "Les apparences sexualisées des femmes [font] donc partie de ce qui [est] "vendu" par leurs employeurs en "échange" d'un emploi [...] et une partie de ce que les employeurs vend[ent] aux clients " ${ }^{19}$. Pourtant, ce que vendent les employeurs suppose un travail invisible et quasi gratuit des femmes qui, pour elles, a un coût certain. 


\section{Références}

Adkins, Lisa (1992). "Sexual work and the employment of women in the service industries". In Mike Savage et Anne Witz (éds), Gender and bureaucracy (pp. 207-228). Oxford: Blackwell.

Alonzo, Philippe (1998). "Les rapports au travail et à l'emploi des caissières de la grande distribution. Des petites stratégies pour une grande vertu». Travail et emploi, 76, 37-51.

Barnier, Louis-Marie (2014). "Protéger une profession en période de crise. Les syndicats des personnels navigants commerciaux face à la "licence européenne" ". Terrains \& travaux, 25 (2), 131-149.

Baum, Thomas (2012). "Working the skies, changing representations of gendered work in the airline industry, 1930-2011». Tourism management, 33, 1185-1194.

Benquet, Marlène (2013). Encaisser! Enquête en immersion dans la grande distribution. Paris: La Découverte.

Champagne, Clara, Ariane Pailhé et Anne Solaz (2015). "Le temps domestique et parental des hommes et des femmes: quels facteurs d'évolutions en 25 ans?». Économie et statistique, 478-480, 209242.

Ciambelli, Patrizia (1996). "La boucle et la marque». Terrain, 27, 115-130.

Connell, Raewyn (2014). "L'organisation sociale de la masculinité». In Masculinités. Enjeux sociaux de l’hégémonie (pp. 59-87), Paris : Amsterdam Éditions.

Desaulniers, Élise (2018). «Donnez-leur des pipes et du steak". In Collectif, Faire partie du monde. Réflexions écoféministes (pp. 43-52). Montréal: Les Éditions du remue-ménage.

Fellay, Angélique (2010). Servir au restaurant: sociologie d'un métier (mé)connu. Thèse de doctorat en sociologie, Université de Lausanne.

Guillaumin, Colette ([1992] 2016). Sexe, race et pratique du pouvoir. L'idée de Nature. Donnemarie-Dontilly: Éditions iXe.

Hall, Elaine J. (1993). «Smiling, deferring, and glirting. Doing gender by giving "good service»". Work and occupations, 20 (4), 452-471.

Hidri Neys, Oumaya (2013). "Le jeu des apparences: piège ou profit?». Travail et Emploi, 134, 75-89.

Hidri, Oumaya et Catherine Louveau (2005). «Travailler son corps: complément du capital scolaire sur le marché du travail? Stratégies d'anticipation d'étudiantes». Travailler, 14 (2), 129-151.

Hochschild, Arlie Russell ([1983] 2017). Le prix des sentiments. Au cour du travail émotionnel. Paris: La Découverte.
Jacquot, Lionel et Ingrid Volery (2019). «Le travail dans la peau. Les figures du corps dans la sociologie du travail contemporaine». La Nouvelle Revue du Travail, 14, [https://journals.openedition.org/nrt/4541] (consulté le 30 mai 2019).

Jeantet, Aurélie (2003). " «À votre service !" La relation de service comme rapport social». Sociologie du travail, 45 (2), 191-209.

Kaufmann, Jean-Claude (1992). La trame conjugale. Analyse du couple par son linge. Paris: Nathan.

Laperrière, Ėve, Karen Messing et Renée Bourbonnais (2010). " "Pour être serveuse, tu dois avoir toute ta tête" : efforts et reconnaissance dans le service de table au Québec». Travailler, 23 (1), 27-57.

Mann, Brenda et James D. Spradley ([1975] 1979). Les bars, les femmes et la culture. Femmes au travail dans un monde d'hommes. Paris: PUF.

Monjaret, Anne (2005). "De l'épingle à l'aiguille». L'Homme, 173, 119-147.

Perramond, Loïc (2015). "Toute ma vie j’ai rêvé... Les uniformes du personnel navigant, entre normes et pratiques, racontés par ceux qui les portent". Modes pratiques. Revue d'histoire du vêtement et de la mode, 1, 218-229.

Rennes, Juliette, Clotilde Lemarchant et Lise Bernard (2019). "Habits de travail». Travail, genre et sociétés, 41 (1), 23-28.

Robert, Camille et Louise Toupin (éds) (2018). Travail invisible. Portraits d'une lutte féministe inachevée. Montréal : Les Éditions du remue-ménage.

Ruault, Lucile (2010). L'étalon de la féminité. Chaussures à talons et marquage des corps de femmes. Mémoire de Master 1, Institut d'études politiques de Lille.

Schütz, Gabrielle (2018). Jeunes, jolies et soustraitées: les hôtesses d'accueil. Paris: La Dispute.

Schütz, Gabrielle (2006). "Hôtesse d'accueil. Les attendus d'un "petit boulot" féminin pour classes moyennes (enquête)». Terrains \& Travaux, 1 (10), 137-156.

Simonet, Maud (2018). Travail gratuit: la nouvelle exploitation. Paris : Éditions Textuel.

Tabet, Paola (1998). La construction sociale de l'inégalité des sexes. Des corps et des outils. Paris: L'Harmattan.

Verdier, Yvonne (1979). Façons de dire, façons de faire. La laveuse, la couturière et la cuisinière. Paris : Gallimard. 